Ebisu Ebisu

Études japonaises Études japonaises

$51 \mid 2014$

Le rapprochement franco-japonais dans l'entre-deuxguerres

\title{
La mission militaire française de l'aéronautique au Japon (1919-1921)
}

\section{Christian Polak}

\section{(2) OpenEdition \\ Journals}

Édition électronique

URL : http://journals.openedition.org/ebisu/1459

DOI : 10.4000/ebisu. 1459

ISSN : 2189-1893

Éditeur :

Institut français de recherche sur le Japon (UMIFRE 19 MAEE-CNRS), Maison franco-japonaise

Référence électronique

Christian Polak, "La mission militaire française de l'aéronautique au Japon (1919-1921) », Ebisu [En

ligne], 51 | 2014, mis en ligne le 01 novembre 2014, consulté le 30 avril 2019. URL : http://

journals.openedition.org/ebisu/1459; DOI : 10.4000/ebisu.1459 


\title{
Document
}

\section{La mission militaire française de l'aéronautique au Japon (1919-1921)}

\author{
Christian POLAK*
}

Dès le début des relations entre la France et le Japon dans les années 1860, le domaine militaire joue un rôle prépondérant. La France apporte au Japon ses technologies et son savoir-faire, dispensé sur place par ses ingénieurs, et fournit le matériel d'armement le plus récent : ainsi, de 1865 à 1876, construction de l'arsenal de Yokosuka ; de 1867 à 1868, première mission militaire française au Japon ; de 1872 à 1880, deuxième mission militaire ; de 1884 à 1889, troisième mission militaire ; de 1886 à 1890, modernisation de la Marine impériale par l'ingénieur Émile Bertin qui conçoit ses nouveaux navires dont certains sont commandés en France. À partir des années 1910, la France fournit des avions de chasse et du matériel aéronautique. En 1918, le Japon lui demande l'envoi d'une mission militaire aéronautique. En reconnaissance de la participation du Japon au premier conflit mondial, Clemenceau envoie aux frais de la République française plus de cinquante ingénieurs et pilotes français qui, de 1919 à 1922, poseront les fondements de l'aviation militaire et de l'industrie aéronautique de l'Archipel. Cette mission est un nouvel exemple, après celui de Yokosuka, d'un transfert de connaissances et de technologies françaises vers le Japon qui peut alors développer une véritable industrie aéronautique moderne.

* Université Meiji, EHESS. 
En 1918, les relations entre la France et le Japon sont régies par l'arrangement - ou l'entente ou encore l'accord franco-japonais - de $1907^{1}$ et reposent sur une amitié entre deux grands personnages, Georges Clemenceau et Saionji Kinmochi 西園寺公望 (1849-1940).

Le Japon s'est intéressé à l'aviation, d'abord aux montgolfières, dès la fin de la période d'Edo. C'est un Français, Yves Paul Gaston Le Prieur (18851963), attaché naval auprès de l'ambassade de France à Tokyo, qui, avec son ami le capitaine Aibara Shirō 相原四郎 (1879-1911), va faire voler le premier planeur, construit de leurs mains en bambou et remorqué sur la place d'Ikenohata à Ueno le 26 décembre 1909, sur une longueur de 130 mètres et à une hauteur de 10 mètres.

Le premier pilote japonais est formé en France, il s'agit de Tokugawa Yoshitoshi 徳川好敏 (1884-1963). Il rapporte de France un Henri-Farman avec lequel il effectue le premier vol d'un avion au Japon le 19 décembre 1910 sur le champ de manœuvre de Yoyogi à Tokyo. Le Henri-Farman, devenu célèbre, se retrouvera dans de nombreuses illustrations ${ }^{2}$.

L’année 1911 s'avère une année charnière pour le Japon : les dirigeants politiques et militaires prennent conscience de l'importance de l'aviation qui exerce un attrait stratégique par la garantie qu'elle offre en matière de défense du pays et de protection de ses colonies, Formose et la Corée. Pour se constituer une force aérienne, le Japon se tourne vers la France qui est à l'avant-garde dans ce domaine, aussi bien sur le plan du matériel que de la pratique. Une mission de la Marine impériale visite la France en 1911,

1. L'arrangement de 1907 garantit le respect du statu quo en Extrême-Orient et de l'intégrité de la Chine : en détail, respect mutuel des possessions de la France et du Japon en Asie (" maintien de la situation respective et des droits territoriaux des deux pays sur le Continent asiatique ") ; pour la France : Indochine et territoire à bail chinois de Guangzhouwan, la zone d'influence sur les provinces du Yunnan, Guangxi et Guangdong ; pour le Japon : Formose et le Liadong et la zone d'influence sur la Mandchourie méridionale, une partie de la Mongolie et du Fujian. La Corée, considérée comme un protectorat, est incluse dans les droits territoriaux, la France acceptant ainsi une colonisation par l'Archipel.

2. Par exemple, pour le $50^{\mathrm{e}}$ anniversaire de ce premier vol d'un avion au Japon, la Poste japonaise sortira un timbre commémoratif avec, dans le ciel, un Henri-Farman aux côtés d'un avion de ligne moderne. 
dirigée par le vice-amiral Shimamura Hayao 島村速雄 (1858-1923) qui visite l'école de pilotage de Maurice Farman (1877-1964) à Buc et fait son baptême de l'air avec le célèbre industriel comme pilote. En décidant de répondre favorablement à cette requête du Japon, la France pense à ses intérêts en Asie, notamment à l'Indochine. Cette coopération entre, de plus, parfaitement dans le cadre de l'arrangement de 1907. Une relation étroite se construit dans ce nouveau domaine de l'aviation, la France va transférer vers le Japon son savoir-faire et ses technologies pendant plus de vingt ans : dès 1912, formation de nouveaux pilotes japonais en France, fourniture d'avions Maurice Farman, Nieuport, Spad et Morane-Saulnier. L'armée de terre installe une première base à Tokorozawa dans le département de Saitama juste au nord de Tokyo, et la Marine sa première base aéronavale près de Yokosuka à Oihama (plus tard Oppama) avec des hydravions Maurice Farman, ensuite avec plusieurs Nieuport commandés en France en 1913.

Par le jeu des alliances, dont l'arrangement de 1907, le Japon déclare la guerre à l'Allemagne le 23 août 1914 ; le lendemain, une escadrille de l'armée de terre, composée de quatre Maurice-Farman et d'un Nieuport s'envole de Tokorozawa vers la Chine, suivie par celle de la Marine composée des quatre hydravions récemment acquis en France. À la suite de nombreux bombardements aériens, la concession allemande de Tsingtao (Qingdao) tombe le 7 novembre.

En 1917, malgré l'effort de guerre, la France accepte de livrer de nouveaux chasseurs au Japon qui seront déployés sur la nouvelle base de Kagamigahara près de Gifu. Au début de 1918, le Japon demande la livraison de seize nouveaux chasseurs. En mai de la même année, la France donne son accord pour la livraison de six Spad, mais lie cet achat à la décision du Japon d'entrer en ligne aux côtés des Alliés, en proposant de créer un front oriental, une intervention militaire en Sibérie dont le Japon devient le principal acteur en déployant, à partir d'août, 73000 soldats sur trois provinces russes d'Extrême-Orient. Le 15 juillet 1918, le Comité de guerre français décide finalement non pas de livrer six Spad, mais d'envoyer trente Salmson. En échange, le Japon accepte que l'organisation de l'aéronautique dans son armée soit réservée à une mission militaire française que le gouvernement français propose le 21 août 1918. Les négociations 


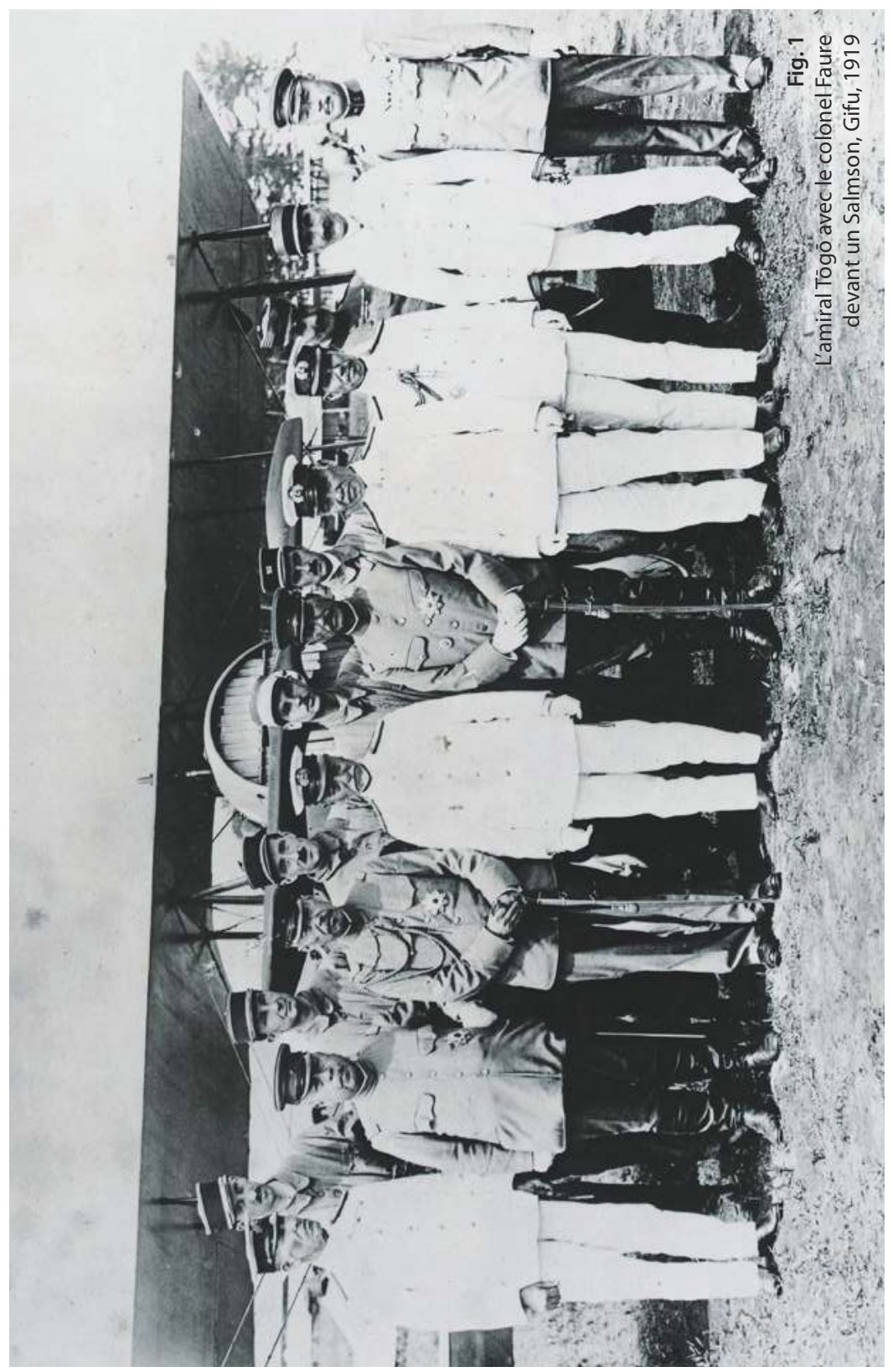


ont lieu à Paris. Georges Clemenceau estime cette affaire de la plus haute importance pour les intérêts français au Japon et en Asie, et supervise les négociations avec l'attaché militaire du Japon, le colonel Nagai Kitaru 永井来 (1877-1934). Une note de Clemenceau, datée du 26 août 1918 et adressée au sous-secrétaire d'État de l'Aéronautique nous donne les précisions suivantes :

"L'Attaché militaire du Japon a fait connaître qu'il acceptait nos propositions... La Mission sera dirigée par le colonel Faure qui choisira ses collaborateurs... Le Gouvernement japonais a en outre manifesté le désir de voir figurer dans cette Mission, à côté du personnel militaire, quelques ingénieurs ou spécialistes des fabrications aéronautiques...»

Plus loin, Clemenceau explique que pour remercier l'action du Japon dans la guerre : «Le Gouvernement Français prendra à sa charge tous les frais de solde, d'entretien et de transport du personnel de la Mission ", ceci à la plus grande surprise du Japon. Le choix du responsable de la mission a été décidé par Clemenceau lui-même. En envoyant cette mission à ses frais, la France écarte les Italiens, les Britanniques et les Américains ; elle espère en retour que le Japon passera de nombreuses commandes à l'industrie française.

Le colonel Jacques-Paul Faure (1869-1924) est officiellement nommé, le 25 août 1918, " chef de la Mission Militaire Française de l'Aéronautique au Japon ». Il s'occupe de la composition de la mission en choisissant des collaborateurs expérimentés avec les meilleures compétences. Il prend comme adjoint le commandant Louis Ragon (1878-1919), fait un choix définitif sur vingt-et-un officiers et sous-officiers dans l'artillerie, le génie, la cavalerie, l'infanterie et l'aéronautique et sur vingt-sept hommes de troupe, ingénieurs et techniciens notamment et bien sûr des pilotes, plusieurs as de la Grande Guerre. Couvrant tous les secteurs de l'aéronautique, la mission comprend au départ cinquante membres au total. Une longue liste de matériel à emporter est aussi préparée, avions Salmson 2A2, divers types de Nieuport, des Spad, des Bréguet ainsi que des ballons Caquot. Faure prévoit un départ de Marseille le 20 novembre 1918 et un retour en France au $1^{\text {er }}$ octobre 1919, envisageant cependant que le personnel de contrôle des fabrications se maintienne plus longtemps jusqu'en 1920 au minimum. Une dizaine de nouveaux instructeurs français se joindront à la mission quelques semaines ou quelques mois plus tard. Le $\mathrm{n}^{\circ} 10$ sur cette liste, le 
lieutenant d'artillerie Roger Poidatz (1894-1976), polytechnicien et pilote de reconnaissance, écrira pendant son séjour le célèbre livre L'Honorable Partie de campagne, paru en 1924 sous le pseudonyme de Thomas Raucat.

La mission s'embarque à Marseille le 24 novembre 1918, treize jours après la signature de l'Armistice qui consacre la victoire des Alliés et de la France. Elle se transborde sur le steamer japonais Yamashiro-maru envoyé à Shanghai par le gouvernement japonais, elle arrive à Nagasaki le 12 janvier 1919, où elle reçoit un accueil triomphal. Elle débarque à Kobe et prendra le train jusqu'à Tokyo où elle arrive le 15 janvier, et à chaque arrêt dans les gares, entonnement de la Marseillaise, discours et cadeaux.

Les plus hautes autorités réservent à Tokyo là encore un accueil triomphal : le directeur de l'Aéronautique Inoue Ikutarō 井上幾太郎 (1872-1965), le ministre de l'Armée de terre, Tanaka Giichi 田中義一 (1864-1929). Ce dernier amplifie les attributions du chef de la mission en lui ouvrant tous les établissements militaires sans aucune réserve. Le 27 janvier la mission est reçue en audience par l'empereur.

Après toutes ces festivités, la mission commence ses travaux. Faure et son adjoint Ragon s'installent dans les locaux du ministère de l'Armée de terre et travaillent en direct avec le ministre Tanaka qui a mis en place la «Commission provisoire d'entraînement aux techniques de l'Aéronautique » présidée par le général Inoue Ikutarō. Un accord signé le 18 janvier avec Faure spécifie les domaines de formation : pilotage, tir, bombardement, télégraphie, photographie, équipements d'usine pour la production et la maintenance.

Après avoir visité en février les installations existantes, les terrains nouveaux proposés pour les développements futurs, la mission est divisée en huit sections de formation réparties sur huit sites :

- Pilotage (combat aérien), base de Kagamigahara près de Gifu ;

- Tir aérien, base d'Arai-machi dans le département de Shizuoka ;

- Bombardement, base de Mikatagahara dans le département de Shizuoka ;

- Observation, à Yotsukaidô dans le département de Chiba ;

- Construction des appareils, base de Tokorozawa, département de Saitama ;

- Fabrication des moteurs, arsenal d'Atsuta à Nagoya ;

- Contrôle, arsenal de Tokyo ;

- Aérostation, base de Tokorozawa. 
La mission reçoit de France la livraison de plusieurs Salmson en caisse qu'elle remonte avec les équipes japonaises. Sont aussi livrés des Nieuport, des Bréguet, des Spad, des Sopwith, des Caudron, un hydravion FBA et un Hanriot.

À la fin du mois d'août 1919, le gouvernement japonais demande la prolongation de la mission jusqu'au 31 mars 1920, prolongation que la France accepte toujours à ses frais.

La mission militaire française d'aéronautique au Japon a ainsi renoué avec la politique de transferts de savoir-faire et de technologies vers le Japon trente ans après le départ de l'ingénieur de la Marine, Émile Bertin (18401924). La mission avait apporté et vendu au total quarante Nieuport, du matériel de reconnaissance, de photographie, de T.S.F., de tir et de bombardement, deux Bréguet 400. Le Japon a acheté dans la foulée quatre-vingt Salsom 2A2, cent avions de chasse Spad 13. Les Nieuport, les Salmson et les Spad vont devenir les chasseurs de référence de l'armée japonaise organisée sur le modèle français : les Japonais achètent les licences du Salmson 2A2, du Nieuport 24C pour les avions de chasse par exemple, et pour les moteurs, celles du V8 Hispano-Suiza et du Lorraine-Dietrich.

Les industriels français vont ensuite vendre des licences, celle du Salmson à Kawasaki Heavy Industriy à l'époque chantiers navals, produits au Japon à 1017 exemplaires, celle du Nieuport 83E2 à la société Nakajima produit à 140 exemplaires, celle du Nieuport 81E2 à Mitsubishi Heavy Industry produit à 60 exemplaires, celle du Nieuport 29C1 à Nakajima produit à 608 exemplaires, celle du moteur Hanriot rotatif à Mitsubishi, etc.

Une grande partie de la mission rentre en France fin mars 1920, mais certains ingénieurs prolongent leur séjour à la demande des Japonais, mais cette fois à leurs frais. Un poste d'attaché militaire pour l'aéronautique est créé en avril 1921 à l'ambassade de France à Tokyo pour poursuivre l'œuvre de la mission Faure.

Une nouvelle mission, plus restreinte, menée par le commandant Marcel Jauneaud (1885-1947) avec deux officiers, arrive en septembre 1921 pour créer une armée de l'air indépendante, une école supérieure de guerre aérienne et un service des études et essais techniques et de fabrications aéronautiques. La première Armée de l'air du Japon voit le jour en 1925 sur le 
modèle français et avec des équipements d'origine française. Une autre mission, dirigée par Antoine de Boysson (1892-1946) avec six autres ingénieurs, arrive au Japon en 1922 et restera un an sur la base de Tokorozawa. Nous avons retrouvé des notes avec photos de l'un des ingénieurs, Georges Metz.

Les grandes entreprises de l'aviation au Japon, Mitsubishi, Nakajima, Kawasaki, inviteront ensuite à leur frais de nombreux ingénieurs français qui travailleront au développement de nouveaux appareils jusqu'en 1935.

\section{Les relations diplomatiques franco-japonaises entre 1919 et 1925}

La Conférence de Washington (1921-1922) entraîne un renforcement discret des relations entre la France et le Japon. Si la plupart des traités ou des alliances, comme l'alliance anglo-japonaise de 1902, sont abrogés, la France et le Japon, de concert, vont maintenir l'arrangement de 1907 et ses clauses secrètes, créant ainsi une atmosphère de rapprochement qui va durer jusqu'en 1925. Les rumeurs officielles ou officieuses évoquent l'éventualité d'une alliance dans le Pacifique entre le deux pays, entente politique et militaire. Le Japon recherchait, devant son isolement, un palliatif à son ancienne alliance anglo-japonaise ; l'Archipel souhaite se tourner vers la France, en lui apportant des assurances de sécurité et de paix dans cette partie du monde où elle ne pouvait entretenir des forces militaires importantes. Afin de concrétiser cet éventuel rapprochement, les deux pays redoublent d'efforts, avec l'ambassadeur Paul Claudel (18681955) à Tokyo, et à Paris, comme ambassadeur du Japon en 1923, un homme politique important, Ishii Kikujirō 石井菊次郎 (1866-1945), ami de Saionji Kinmochi. La France envoie le maréchal Joseph Joffre (18521931) au Japon en 1922, puis le gouverneur de l'Indochine Martial Merlin (1860-1935) en 1924. Le Japon envoie à son tour une mission en Indochine. Certes, les deux gouvernements se sont penchés sur cette possibilité d'alliance, mais ni l'un ni l'autre ne va l'évoquer ou la proposer concrètement. Le contentieux économique entre l'Indochine et le Japon fut certainement la principale raison qui empêcha toute négociation officielle de ce " projet ". 
Paul Claudel utilisait l'influence de son ami Alexis Léger (1887-1975), ancien secrétaire à Pékin (1916-1921), l'un des négociateurs à la Conférence du désarmement de Washington, puis à partir de 1922, chef de cabinet du ministre des Affaires étrangères, Aristide Briand (1862-1932). Léger appuyait en privé toutes les propositions de Claudel (dont la visite de Joffre et de Merlin). Briand et le président du Conseil Raymond Poincaré (18601934) ont ainsi soutenu ces deux projets de visite au Japon.

Dans ses dépêches, Claudel utilise souvent le terme de "rapprochement " afin de ne pas effrayer le gouvernement français soucieux de rétablir de bonnes relations avec les États-Unis et la Grande-Bretagne. Claudel compare le Japon à un "Robinson international ", isolé dans son coin, et fait remarquer que l'arrangement franco-japonais de 1907 constitue, surtout en cas de conflit, une véritable alliance militaire avec le Japon et ce malgré les accords de Washington puisque ni la France ni le Japon n'avaient pris l'initiative de l'annuler. La presse japonaise évoquera souvent l'imminence de la signature d'une nouvelle alliance franco-japonaise en précisant parfois qu'elle sera militaire. Poincaré et Briand sont restés sur le terme " rapprochement " plutôt que de s'avancer sur une " alliance militaire " qui ferait double emploi avec l'arrangement de 1907. Une campagne de presse américaine virulente contre le resserrement des relations entre la France et le Japon freine aussi l'enthousiasme du gouvernement français. Ce " projet " disparaît définitivement avec l'absence du Japon de son initiateur français Claudel pendant plus de treize mois entre 1924 et 1925, absence qui amènera une grande déception chez les partisans japonais, notamment le maréchal Uehara Yūsaku 上原勇作 (1879-1933).

Sources: se rapporter au texte de l'auteur paru dans Sabre et Pinceau, par d'autres Français au Japon (1872-1960), publication de la Chambre de commerce et d'industrie française du Japon, Tokyo, 2005, pages 76 à 151, ainsi qu'à sa thèse de doctorat soutenue à l'université Hitotsubashi en 1980 et intitulée La politique française en Extrême-Orient et le Japon de 1914 à 1925. Pour les transferts de technologies de la France vers le Japon dans les dernières années du shogunat: Soie et Lumières. L'âge d'or des échanges franco-japonais, des origines aux années 1950, publication de la Chambre de commerce et d'industrie française du Japon, Tokyo, 2001 ; voir aussi Lys et Canon, Images et correspondances retrouvées, 1860-1900, idem, Tokyo, 2013. 


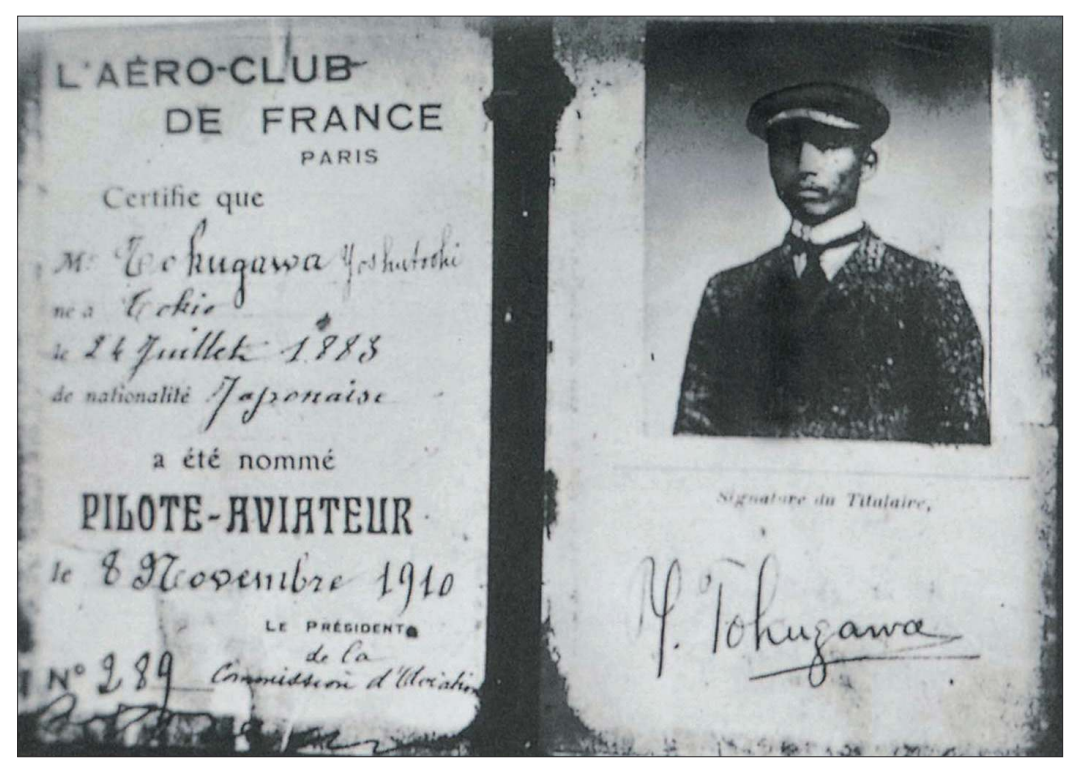

Fig. 2

Le brevet de pilote

de Tokugawa Yoshitoshi

\section{Ci-contre}

Fig. 3

Un Farman à Oihama

(Oppama)

Fig. 4 Le premier simulateur de vol sur la base de Kagamigahara, près de Gifu 

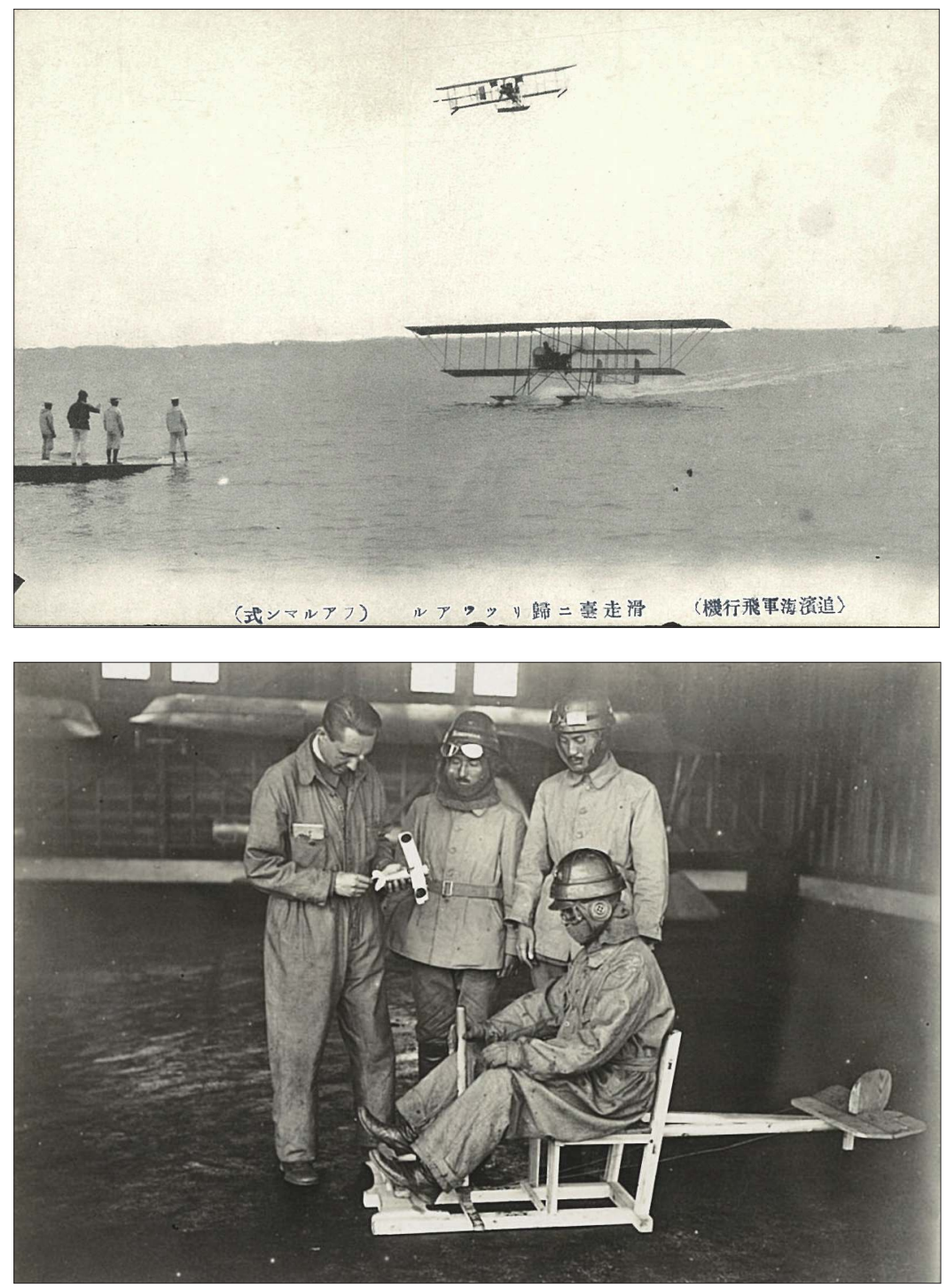


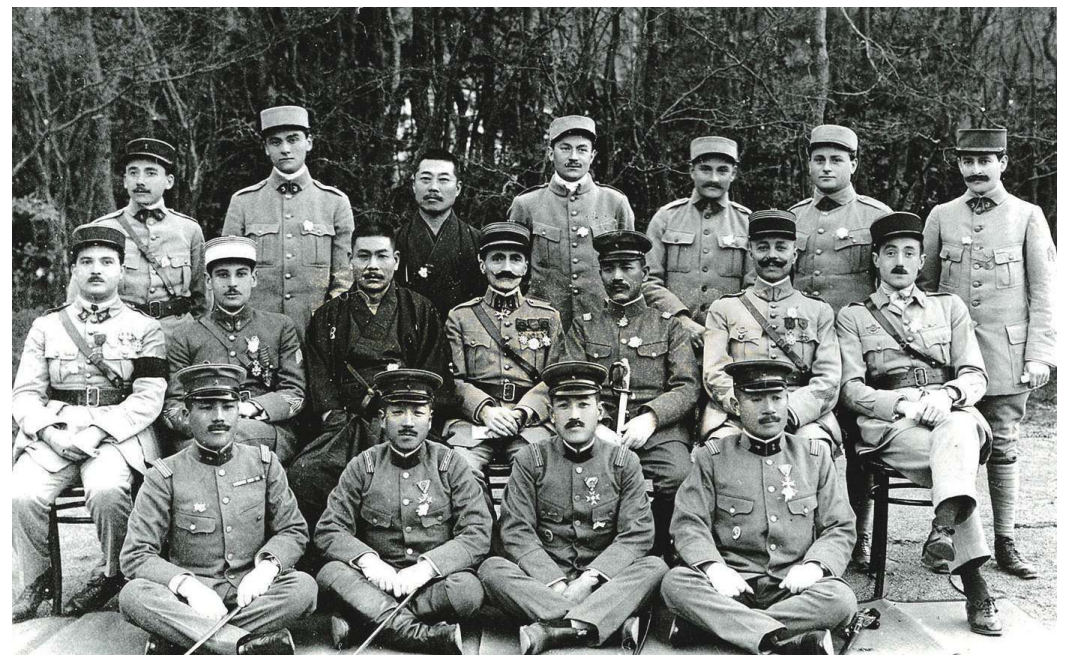

Fig. 5

Le colonel Faure au centre avec sa moustache, et des officiers japonais à Tokyo en 1919

Fig. 6

Le tampon de la mission

Fig. 7

Le colonel Faure devant un Salmson, Gifu, 1919

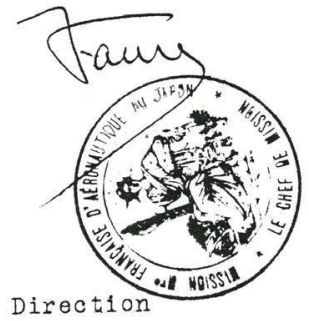

Fig. 8 ci-contre État nominatif du personnel de la mission, au départ de Paris

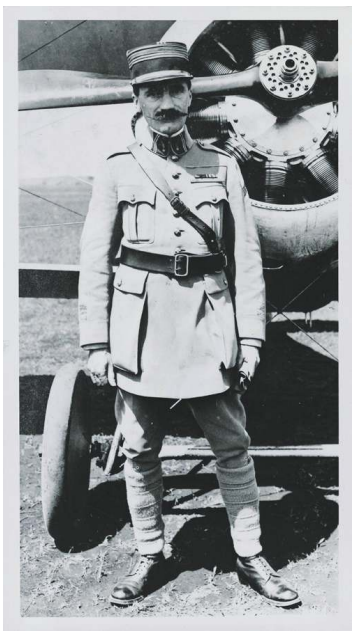

Fig. 9 et 10 pages suivantes

État nominatif du

personnel de la mission,

en mai 1919

État nominatif du

personnel de la mission

(p. 2), en juin 1919 


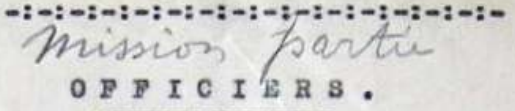

\section{Colonel}

Cher a'Bees 6ron

Cher a 'sBcadron

Cher de Bata112on

Chef a 'Bsesdron

Ileutenant

Ileutenant

Ilentemant

Lieutenant

Lieutenant

S/Ileutenant

S/Ileutenant

s/Ieutenant

s/Ileutenent à titre Ilostig

s/Lieutenant lo 22 ivr11 1919

s/Ilentenant

s/Ileutenant

s/Ileutenant

s/Lleutenant

S/Lieutenant \& titre Pletis

le 15 Thrs 2919
II 0 if $\mathrm{s}$

$\mathrm{P}$ R B E 0 if $\mathrm{S}$

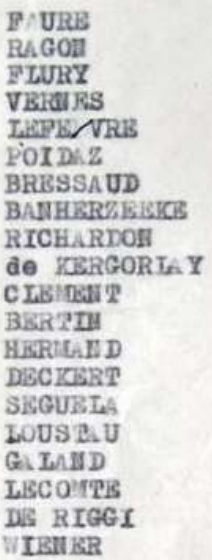

JOSSIT off; $a^{\prime}$ dn. $2^{\circ}$ Classe nomb offioler $a^{\circ}$ a. Prinelpal à titre fletif le 11 Avril 1919.

VUARIII

P IIRR III

VIBत्र ISSB

BOUCHOS

HUGUis?

SIRRIST

Laront

RCOUBR $X$
France, Joseph

antolne, Joannds

Mtiohel, Henri, Marius Jenn
Ja oques, Panl

Loule, a ugaete

Zavlex, liario, antolna

Gabrle1, Jacques

Chrries

Jenn, Iouis

Ibert

Bugène, Jrenço1s

George日

Llbert

plerre.

Froncois

Paul, Henr 1

Zdoverd, llaroel

Btienne

Joseph, Henr1

Alfred, Charles

Luei en, Bdmona

Bdoasrd, Ranpl

Pleyaond, Plerre, F.
S/Ileutemant

s/Ileutenant

S/Ilentenant aे titre flotip

Ileutenant

Il eut enint

S/Lleutenant nomme ì titre flotif 20 9 avril 1919

HO:T TSS DE TROUPE

Adjudant

Bergent
4

20 en Instance de aupart
( 1 Contrồ eur

1 Sergent méentelen 


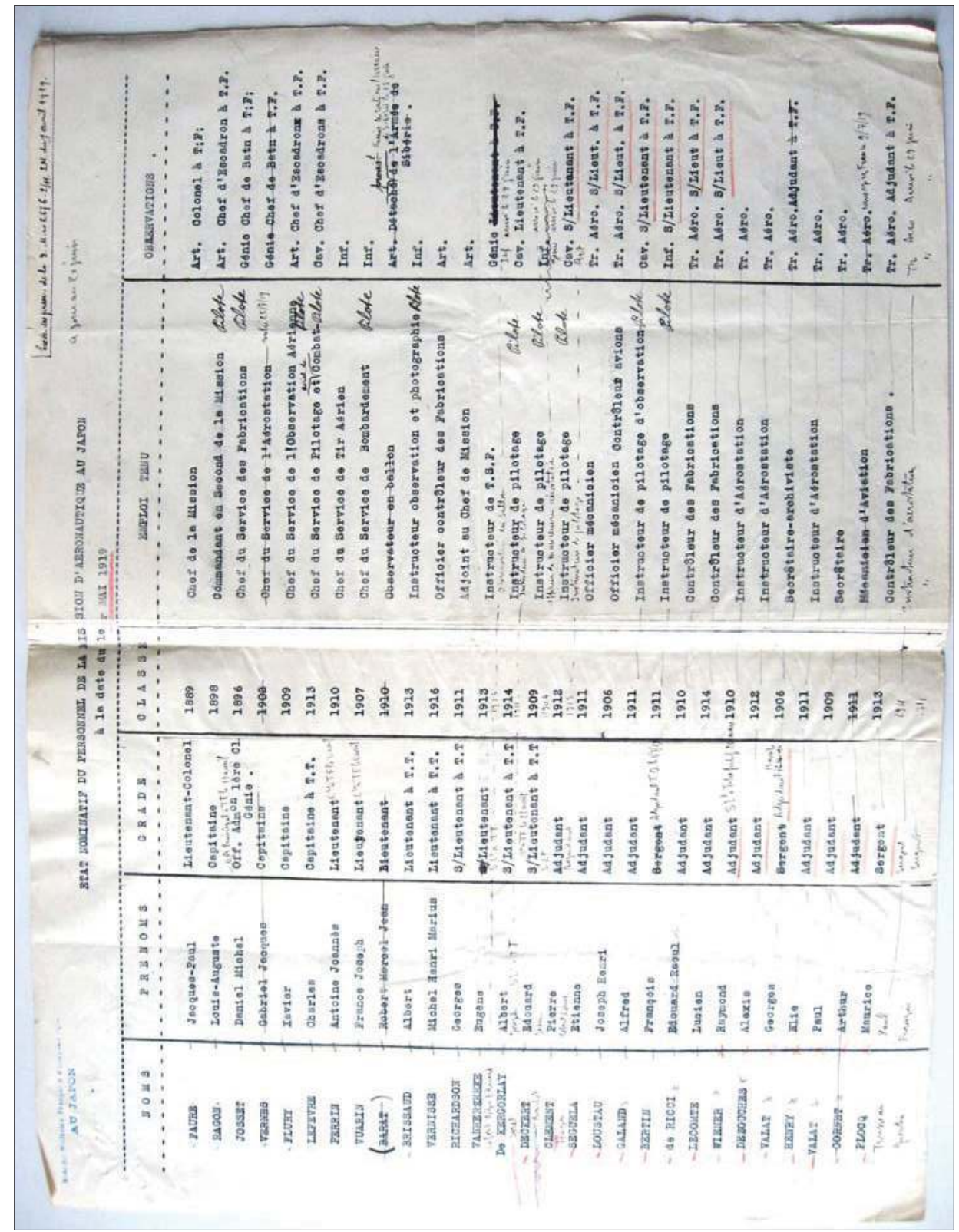




$$
\begin{aligned}
& \text { एं बिं बं } \\
& \text { का को का }
\end{aligned}
$$

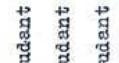

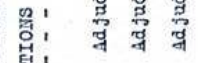

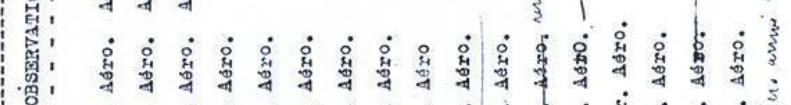

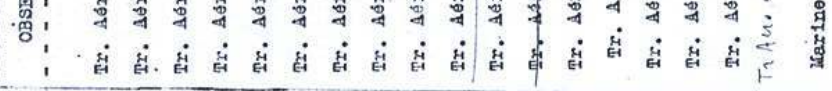
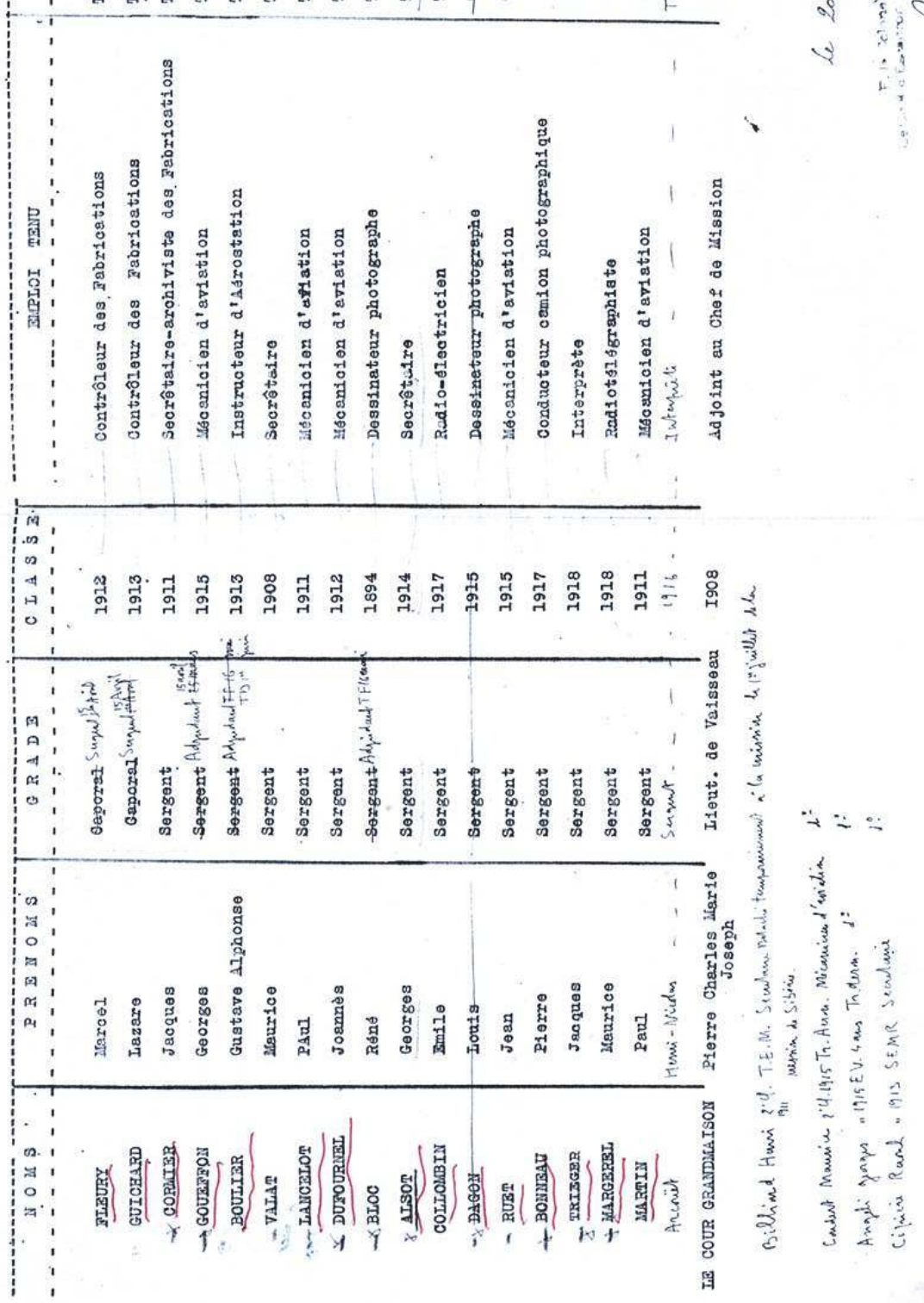


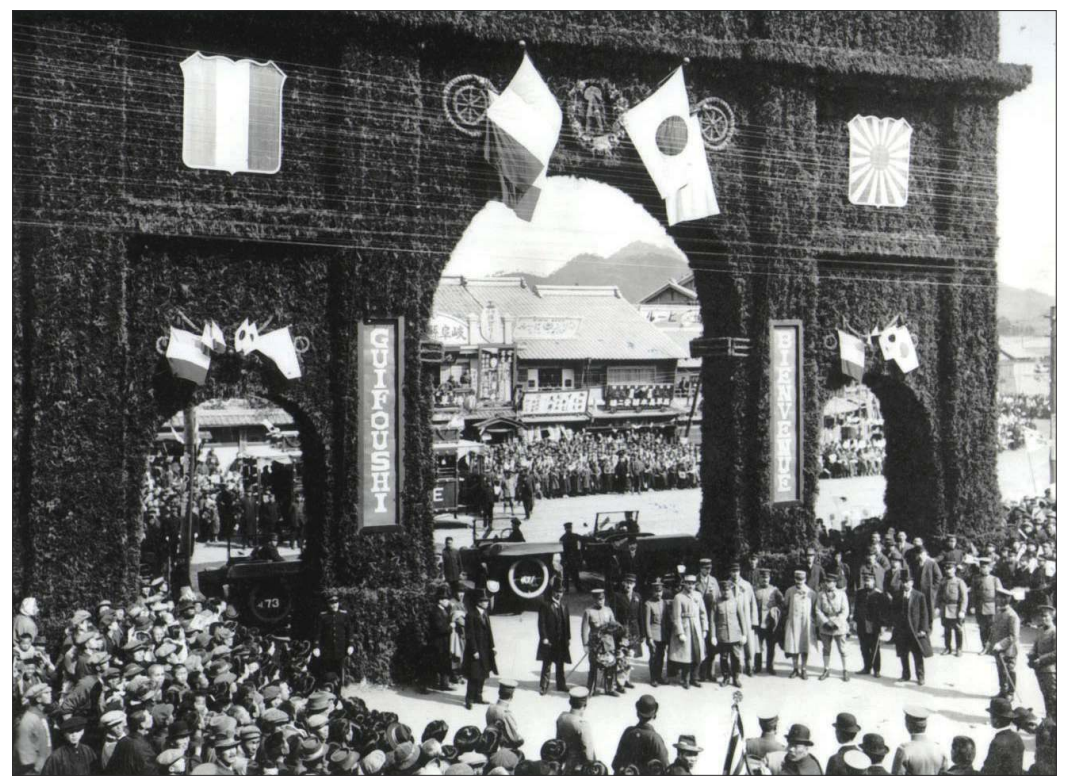

Fig. 11

L'accueil triomphal des membres de la mission à Gifu , avec un arc de triomphe construit pour l'occasion, février 1919

Fig. 12 Les membres de la mission reçus à Tokyo à leur arrivée, en janvier 1919

Fig. 13 Réception de la mission par les militaires japonais à Tokyo, en 1919 

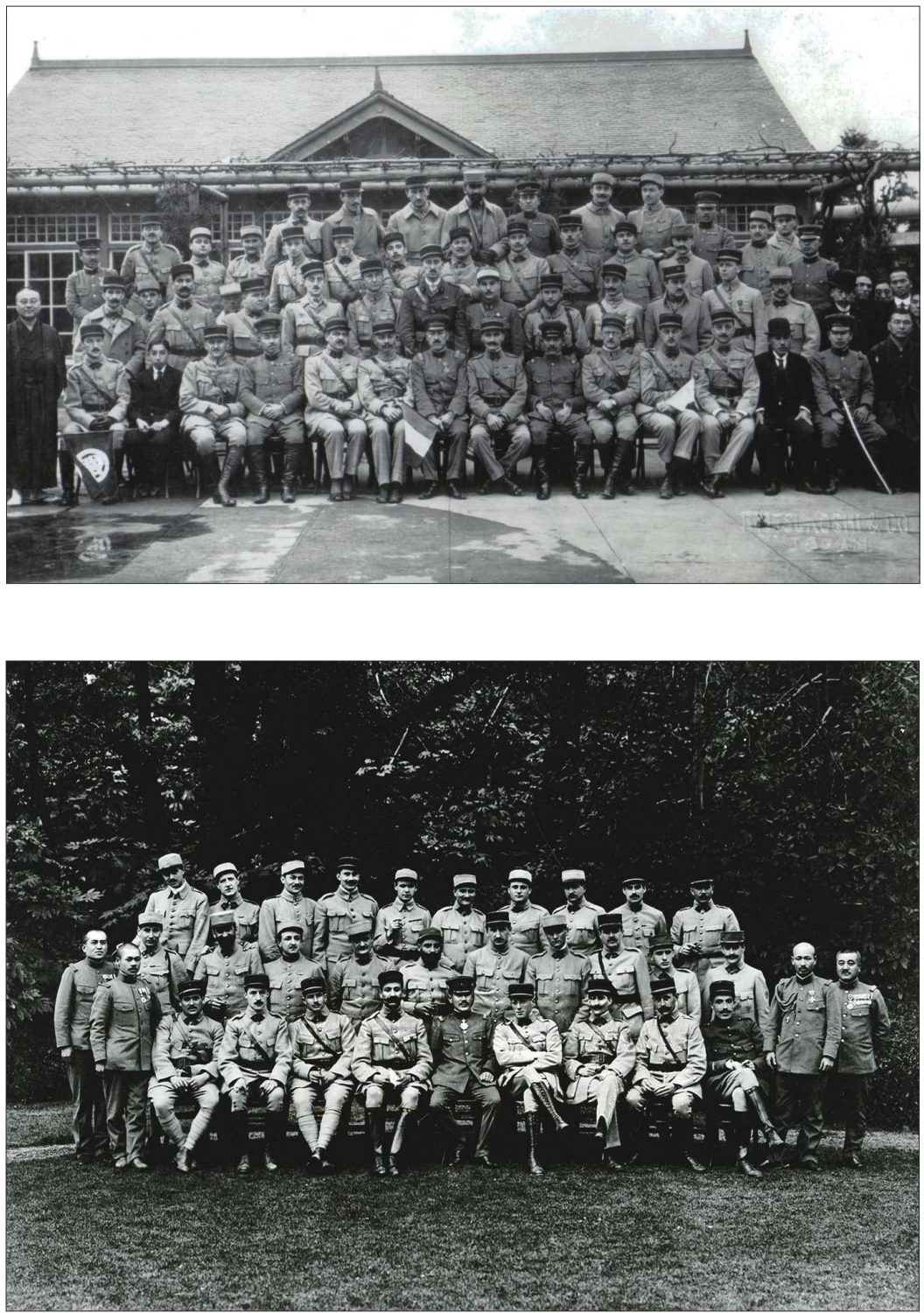


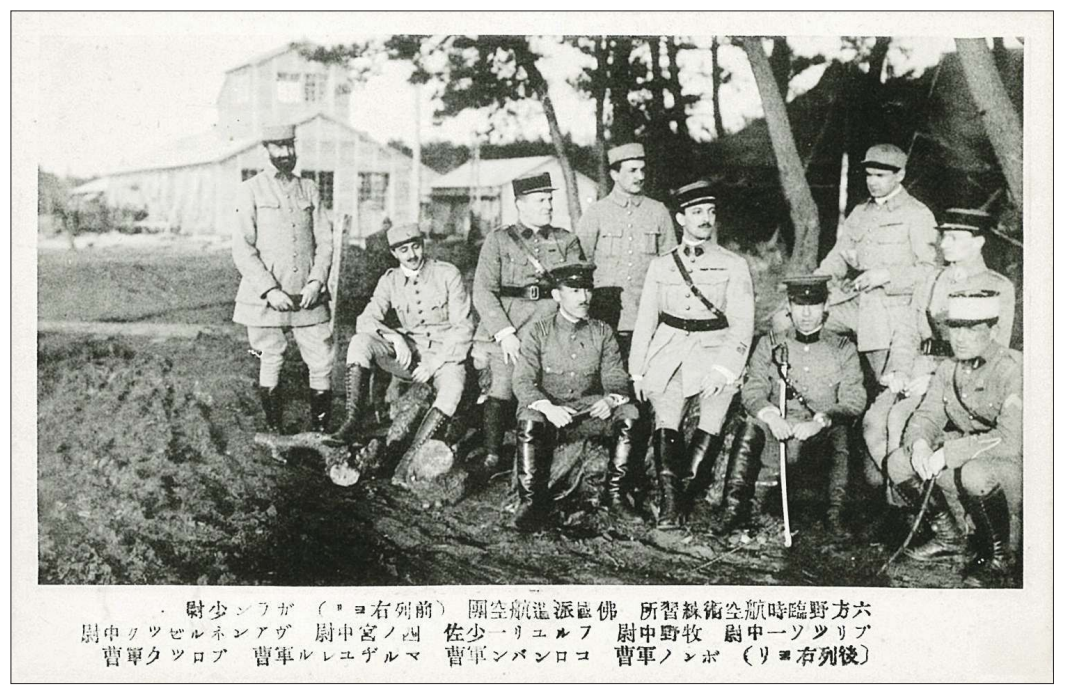




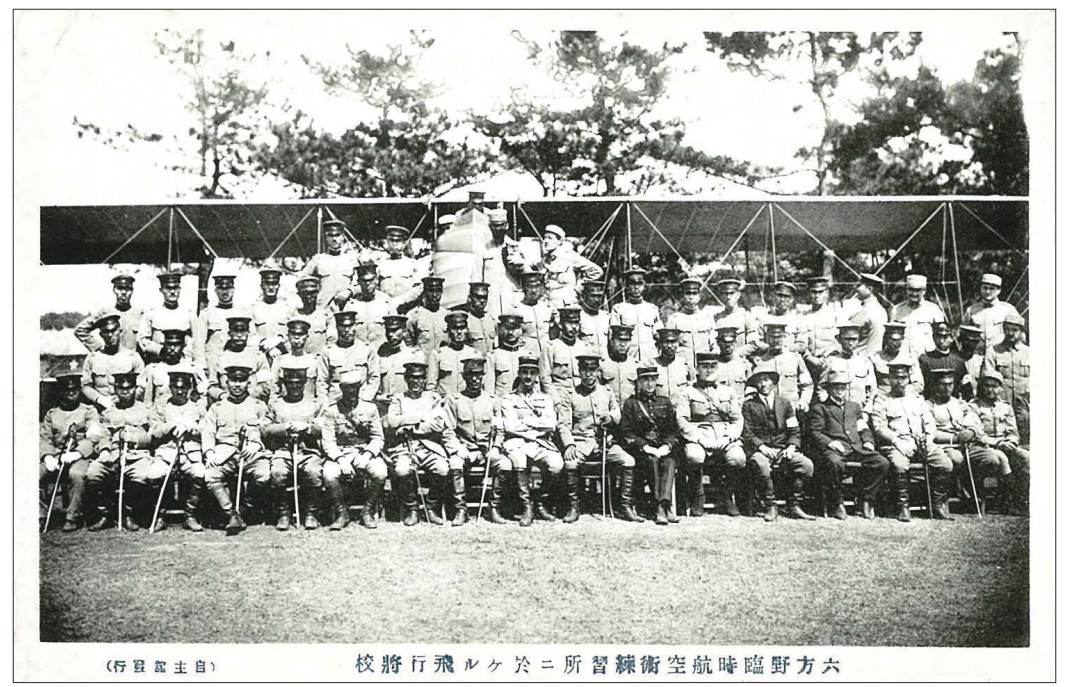

Fig. 14 et 15 en double page Membres de la mission à Roppōno avec leurs élèves japonais, 1919 


\section{Paul Clandel}

Sembausaderex the Franer
GEORGES METZ

INGENIEUH A.\&. N

SERVICE TECHNIQUE

AEROMAUTIOUE MILITAIRE JAPONAISE

La Mission était incorporeé à I'Ecole d'Aviation Militaire de Tokarogawa. Mais elle restait sous la tutelle de I'Ambassadour de France Poul CLAUDEL, la liaison elant assuree par I'Attache' Commercial de I'Ambassade, Mr MAlRE En effet, l'abjectif e'tait de vendre du mate'riel.

De's 1890 , he Japon avait achete' en France des ballons caprifs et des dirigeablest, puis apre's la Guerre des avions: Nieuport er SPAD pour la chasse, SALMSON pour ta reconnaissance, FARMAN 50 er 60 pour le bombardement.

C'était un Gadz'Arts. PAGNY (Ch.02) qui representsit les Societe's frangaives deeronantique j Tokyo.

Voici quelquar membres de la mission devant son e'tablissement

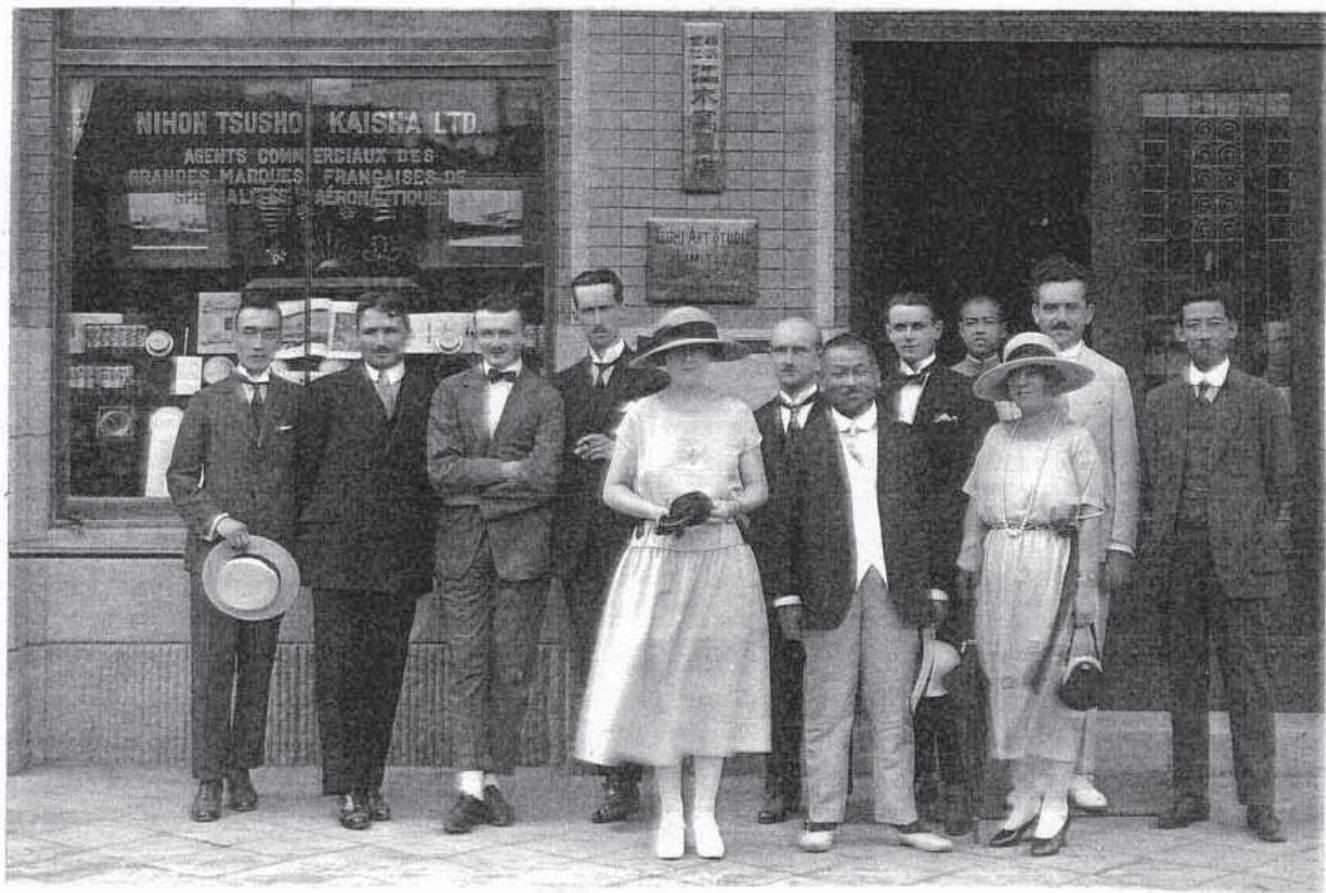




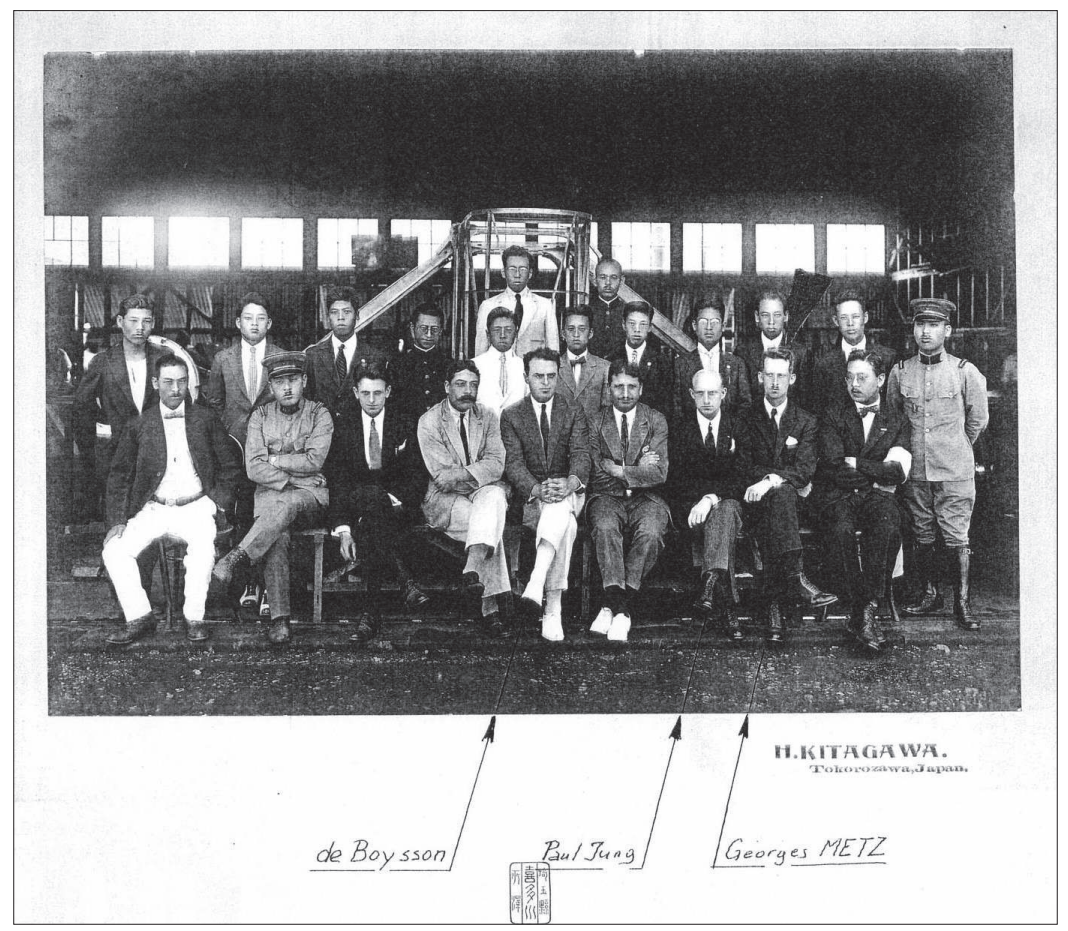

Fig. 17

Mission de Boysson

Fig. 16 ci-contre

Membres de la mission de Boysson 
